\title{
An Assessment of Math Proficiency Among High School Students in Northwest Florida
}

\author{
Josaphat A. Uvah, Kuiyuan Li \\ University of West Florida, Pensacola, USA
}

\begin{abstract}
In this study, we assess the group performance of 970 high school students in mathematics. We use data from the American mathematics competitions (AMC) tests administered at the University of West Florida (UWF) for five consecutive years, 2007-2011, to assess the proficiency level of the participants as a group. The main aim is to identify areas of strength, and more importantly, weaknesses in high school mathematics for the group. A secondary objective is to assess the college readiness of the students in the area of mathematics. Aside from identifying some factors known to affect students' performance at the high school levels, we offer a range of suggestions on individual and institutional remedies that could be implemented in the 11th and 12th grades when most students take the standardized tests in preparation for entry into institutions of higher education. Other recommendations may help students with college readiness in mathematics. Although the students were drawn from two counties-Escambia and Santa Rosa, the results of the study may be useful throughout Northwest Florida and beyond. The results of the study could also be used to guide grade teachers to place emphases on some topics in the mathematics high school curriculum.
\end{abstract}

Keywords: assessment, high school math proficiency, college readiness non-standardized tests

\section{The American Mathematics Competitions (AMC) Tests}

The AMC is an international project of the renowned Mathematical Association of America (MAA). Three annual competitions are held concurrently: the AMC-8 for grades lower than 9th, the AMC-10 for 9th and 10th grades, and the AMC-12 for 11th and 12th grades students. In February, 2011, the AMC-12 and the AMC-10 were the 62nd and the 12th annual competitions, respectively. Each competition comprises 25 questions of varying degrees of difficulty. The questions span the spectrum of pre-calculus mathematics areas ranging from topics traditionally taught in grade school to advanced problems requiring out-of-the-box thinking and fairly complex set-up and manipulation. The competitions are aimed at strengthening the mathematical capabilities of the youth and spurring their interest in mathematics through solving challenging problems. As a byproduct, students may develop further interest in the mathematical sciences to pursue studies in science, technology, engineering, and mathematics (STEM).

\section{Participants for the Study}

Beginning in 2006, during the Fall Semester, the Department of Mathematics and Statistics at the University of West Florida (UWF) sends letters of notice and invitation to heads of Mathematics Department at

Josaphat A. Uvah, Ph.D., professor, Department of Mathematics \& Statistics, University of West Florida.

Kuiyuan Li, Ph.D., distinguished university professor, Department of Mathematics \& Statistics, University of West Florida. 
all high schools that are within 30 miles of the UWF-AMC test center. The target schools are in Escambia and Santa Rosa counties in the Northwest Florida panhandle. During the months that follow, the AMC Test Committee in the department engages authorities at the invited schools by telephone, to determine their interest and availability to participate. Thus, participation by schools is on a voluntary basis. The enquiry reveals that most schools volunteer to bring their students to the competitions which have no restrictions on who could participate. As per the competition rules, 9th and 10th grades students who are under 17.5 years of age may only engage the AMC-10 competition while 11th and 12th graders take the AMC-12 test. In this study, we examine students' performance on portions of the AMC-10 and the AMC-12 tests that we identified, to assess their proficiency in the high school mathematics curriculum. We also examine the students' performance on problems that may give an indication of their readiness for college-level mathematics courses.

We collected data on 970 students for this study from 2007 to 2011. These students from over nine high schools volunteered to take the AMC tests at the UWF-AMC test site. The number of participating schools rose from five in 2007 to nine in 2011. Similarly, the number of students increased from 145 to 283 during the same period. Table 1 below gives a year-by-year breakdown of number of participants in the two AMC tests.

Table 1

Number of Students by Year

\begin{tabular}{llll}
\hline Year & AMC-10 & AMC-12 & Total \\
\hline 2007 & 79 & 72 & 151 \\
2008 & 92 & 53 & 145 \\
2009 & 78 & 67 & 145 \\
2010 & 117 & 129 & 246 \\
2011 & 121 & 162 & 283 \\
Total & 487 & 483 & 970 \\
\hline
\end{tabular}

Testing/Grading

For the years in question, on the third Wednesday in February, students from participating schools arrived at the Pensacola campus of UWF shortly after 8 a.m.. After addressing logistical issues, we administered it for 75 minutes. The tests were sent to the MAA for computerized grading. The students' responses were scored according the established and publicized grading scheme that awarded 6 points for each correct response, 2.5 points for each number that the student left blank, and no points for each incorrect answer. The MAA graded the tests and provided each test center a detailed summary of group as well as individual student's performance on each question.

\section{Math Preparation of the Students}

In order to determine the type of courses that participants in the AMC tests take at the various grade levels, we developed and administered a survey to the group of students who took the AMC tests in 2009. The survey identified a participant's school, current grade level, and mathematics courses that they either had completed or were taking at the time, and the area of study that they intended to pursue in college. We pointedly inquired about Algebra I, Algebra II, Geometry, Pre-Calculus Algebra, Financial Mathematics, Statistics, and Differential and Integral Calculus. A blank line was provided for participants to write-in any courses that we did not name. In our attempt to receive unadulterated responses, the survey was anonymous as the participants did not provide any personal identifying information. Although all participants had taken Algebra I, 
approximately one-third of 9th graders had not taken Algebra II. In addition to Algebra I, all 10th graders had taken Algebra II and Geometry, but a third of them had not taken any mathematics courses beyond Geometry. Similarly, all 11th and 12th graders had taken Algebra I, Algebra II, and Geometry, but only $50 \%$ of them had taken Trigonometry and $27 \%$ of them had a Statistics course. In Table 2, where we summarize the mathematics background of participants in 2009, we note that we grouped College Algebra and Pre-Calculus Algebra together since the difference between the two courses may not be significant at the high school level. We note, in addition, that some schools and colleges offer a pre-calculus course that includes trigonometry.

Table 2

Courses Taken at Various Grade Levels in 2009

\begin{tabular}{lllll}
\hline Courses & 9 th grade $(N=42)$ & 10 th grade $(N=27)$ & 11 th grade $(N=44)$ & 12 th grade $(N=23)$ \\
\hline Geometry & 37 & 27 & 44 & 23 \\
Pre-Calculus Algebra & 0 & 16 & 38 & 22 \\
Trigonometry & 0 & 7 & 27 & 7 \\
Statistics & 0 & 4 & 16 & 2 \\
Calculus & 0 & 2 & 25 & 21 \\
\hline
\end{tabular}

Scope of the Study

The study focused on the students who participated in the tests as a group rather than as individuals. Furthermore, we did not identify the various schools that participated in the tests neither did we differentiate between, or compare the performance of students, by school district. In essence, we studied the group of students as a whole, thereby, limiting our ability to provide information that is particular to a given student, a particular school, or a specific district. While not arguing that our sample was purely random, we maintain that the observations were consistent from year to year for five consecutive years. We were, therefore, convinced that the trends we noted do exist and that the results are indeed valid. Perhaps, another limiting factor in interpreting our results lies in the absence of correlating students' performance with the courses they had taken.

\section{Study Design and Methodology}

\section{The Florida Mathematics Standards and the AMC Tests}

The Florida Department of Education has a long list of topics to be covered in various areas of the mathematics curriculum for high school. Until recently, the minimum requirement in mathematics for high school graduation was Algebra I and Geometry (Engaging Latino Communities in Education [ENLACE] Florida, 2009). The low threshold in mathematics for high school graduation is bound to affect the curriculum that school districts provide for their students. However, most high schools offer more advanced mathematics courses to include the Differential and Integral Calculus as can be seen from the 2009 survey. On the other hand, the AMC tests feature challenging word problems that span the spectrum of pre-calculus mathematics. Test questions demand a thorough understanding of one or more topics often drawn from a number of areas of mathematics. In many cases, problems involved applications of the concepts rather than basic or routine manipulations of mathematical quantities.

\section{Coding of the Problems}

First, we examined each test to classify the topics according to broad areas of mathematics: (a) algebra including arithmetic; (b) geometry, to include rudimentary aspects of trigonometry; (c) discrete structures and 
mathematical logic, and (d) statistics. We then coded the problems by level of difficulty or sophistication as follows:

1. Regular strength: Involving one main concept or one topic in one distinct area other than an advanced topic;

2. Medium strength: Concerning no more than two distinct areas of study or two distant topics within one distinct area of study, to exclude advanced topics;

3. High strength: Involving advanced applications of more than one topic or involving more than two topics.

For a given year, at least 10 but no more than 14 of the problems were identical on the AMC-10 and AMC-12 tests. Almost all problems on the AMC-10 tests were considered to be regular or medium strength. Table 3 summarizes the coding of problems for AMC-10 for the five years in question:

Table 3

Distribution of Test Questions in AMC-10

\begin{tabular}{lll}
\hline Year & Regular & Medium \\
\hline 2007 & $1,2,3,4,5,6,7,8,9,12,14,16,17$ & $10,11,13,15,18,19,20,21,22,23,24,25$ \\
2008 & $1,2,3,4,5,7,8,9,10,12$ & $6,11,13,14,15,16,17,18,19,20,21,22,23,24,25$ \\
2009 & $1,2,3,4,5,6,7,8,9,10,11,15$ & $12,13,14,16,17,18,19,20,21,22,23,24,25$ \\
2010 & $1,2,3,4,5,6,7,8,9,10,12,15$ & $11,13,14,16,17,18,19,20,21,22,23,24,25$ \\
2011 & $1,2,3,4,5,6,7,8,10,11,15,18$ & $9,12,13,14,16,17,19,20,21,22,23,24,25$ \\
\hline
\end{tabular}

The AMC-12 tests featured many high strength problems, thereby, distinguishing these from the AMC-10. Approximately $63 \%$ of the questions that were common on both tests were of regular strength. In addition, topics, such as "expected value in statistics" and "logarithms and geometric series in algebra" appeared on the AMC-12 tests exclusively. In Table 4 below, we summarize the coding of AMC-12 tests.

Table 4

Distribution of Test Questions in AMC-12

\begin{tabular}{lll}
\hline Year Regular & Medium & High \\
\hline $2007{ }^{*} 1,{ }^{*} 2,{ }^{*} 3,4,{ }^{*} 5,{ }^{*} 8,9,{ }^{*} 10,11,{ }^{*} 12$ & $6,{ }^{*} 7,13,{ }^{*} 14,16,19,{ }^{*} 24$ & $15,17,18,20,21,22,23,25$ \\
$2008{ }^{*} 1,{ }^{*} 2,{ }^{*} 3,4,{ }^{*} 5,6,{ }^{*} 7,{ }^{*} 8,{ }^{*} 9,{ }^{*} 10,11$ & ${ }^{*} 12,13,14,{ }^{*} 16,18,19,{ }^{*} 20$ & $15,17,21,22,23,24,25$ \\
$2009{ }^{*} 1,{ }^{*} 2,3,{ }^{*} 4,{ }^{*} 5,{ }^{*} 6,{ }^{*} 7,{ }^{*} 8,{ }^{*} 11$ & $9,{ }^{*} 10,13,{ }^{*} 14,15,16,{ }^{*} 17$ & $12,18,19,20,21,22,23,24,25$ \\
$2010{ }^{*} 1,2,{ }^{*} 3,{ }^{*} 4,{ }^{*} 5,{ }^{*} 6,{ }^{*} 7,{ }^{*} 8,{ }^{*} 10$ & $9,{ }^{*} 11,12,13,14,{ }^{*} 16,{ }^{*} 17,{ }^{*} 19,{ }^{*} 21$ & $15,18,20,22,23,24,25$ \\
$2011{ }^{*} 1,{ }^{*} 2,{ }^{*} 3,{ }^{*} 4,5,10,11$ & $6,7,{ }^{*} 8,{ }^{*} 9,{ }^{*} 12,{ }^{*} 13,14,15,{ }^{*} 16,17,{ }^{*} 18,{ }^{*} 19$ & $20,21,22,23,24,25$ \\
\hline
\end{tabular}

Note. ${ }^{*}$ indicates that the problem appeared on both AMC tests for the corresponding year.

In the main questions that were classified as regular strength were deemed to be drawn from topics, types, and levels of sophistication closest to problems that are typically discussed in a classroom setting and/or placed on class tests. Although the topics from which problems that we classified as medium strength or high strength problems were drawn are also found in the Florida mathematics curriculum for Grades 9-12, each problem of medium strength involved a combination of distantly related topics and a level of sophistication that was deemed to be beyond the scope of routine classroom considerations. We took these factors into consideration to arrive at a set of problems to be used in a more focused analysis in order to assess students' performance in mathematics at the high school level. While the distinction between regular strength and medium strength problems may be slight, the difference between these two and the high strength problems was remarkably 
pronounced. The high strength problems were indeed challenging, because they were intricately crafted from topics we considered to be advanced for high school mathematics.

\section{Analyses and Results}

\section{Selecting Problems for the Study and Determining Proficiency}

We isolated the regular strength problems exclusively to assess students' group performance on the AMC-10 and AMC-12 separately. Next, we weighed the possibility that some students who took the AMC tests may had seized the opportunity of participating in the tests as a golden "escape" from the routine of school. Moreover, we also reasoned that the grading scheme for the tests did not encourage students to guess the correct answer to a given problem since an incorrect response attracted zero points but leaving a blank for a response, an indication of "not sure," was rewarded with 2.5 points. For the purpose of this study, we concerned ourselves with correct responses on the regular strength questions only. Consequently, we judged that groups were proficient on topics or problems on which at least $40 \%$ of the students chose correct responses. Thus, we deemed the group performance on a problem to be deficient if less than $40 \%$ of responses were correct. For this study, blank answers were counted among the incorrect responses. For the AMC-10 and AMC-12 tests, we analyzed students' performance on the selected problems on a year-by-year basis.

\section{Performance on the AMC-10 Tests}

During the five years included in this study, a total of 487 students took the AMC-10 tests. Figure 1 below gives a breakdown of students' performance (in percentages of correct responses) on each problem in the various years in two formats.

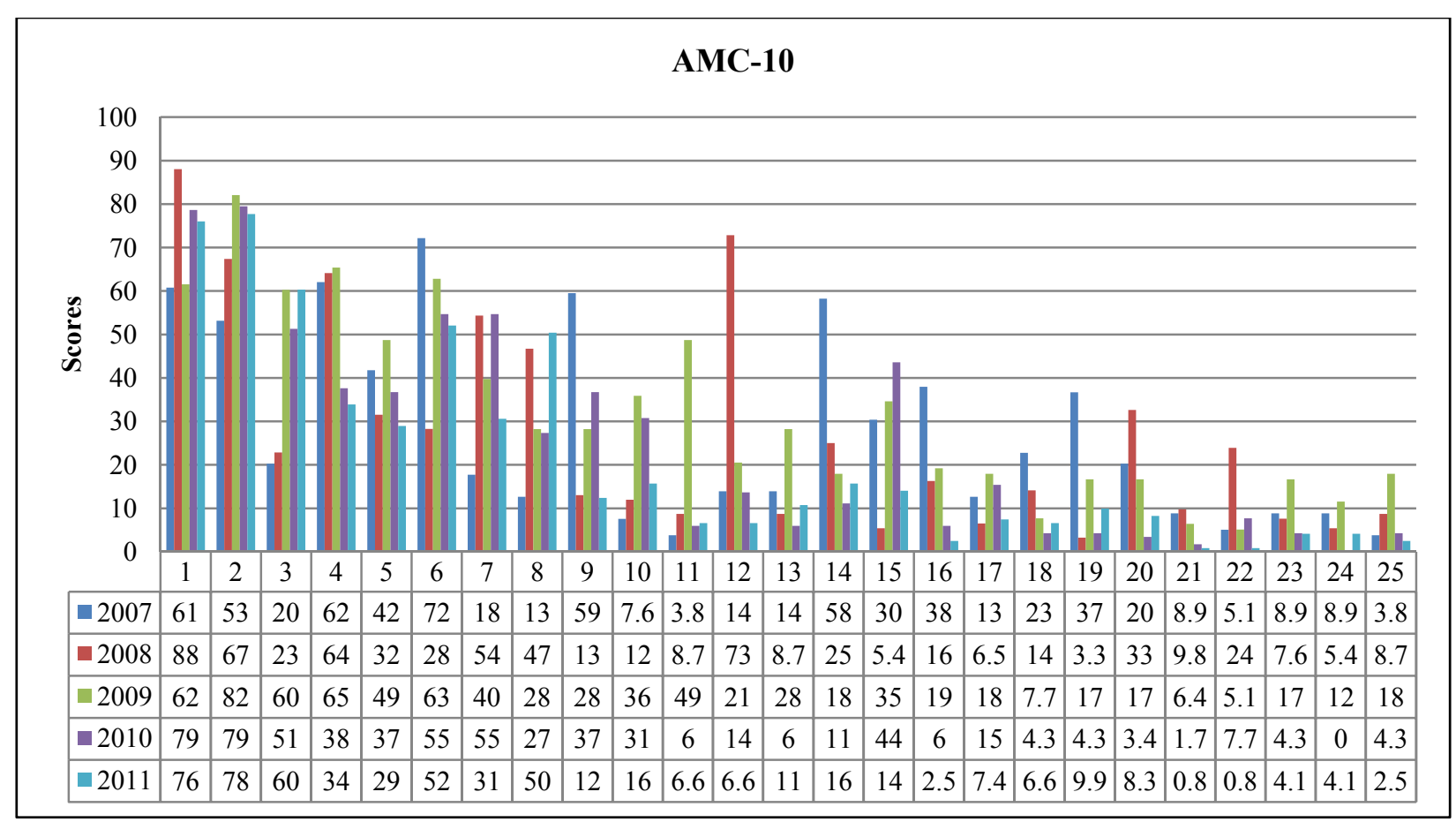

Figure 1. Percentages of correct responses.

We then examined data of students' performance to identify areas of strength and weakness, one year at a time (see Figure 2). We summarize our determinations for the AMC tests in Tables 5a-5e. 


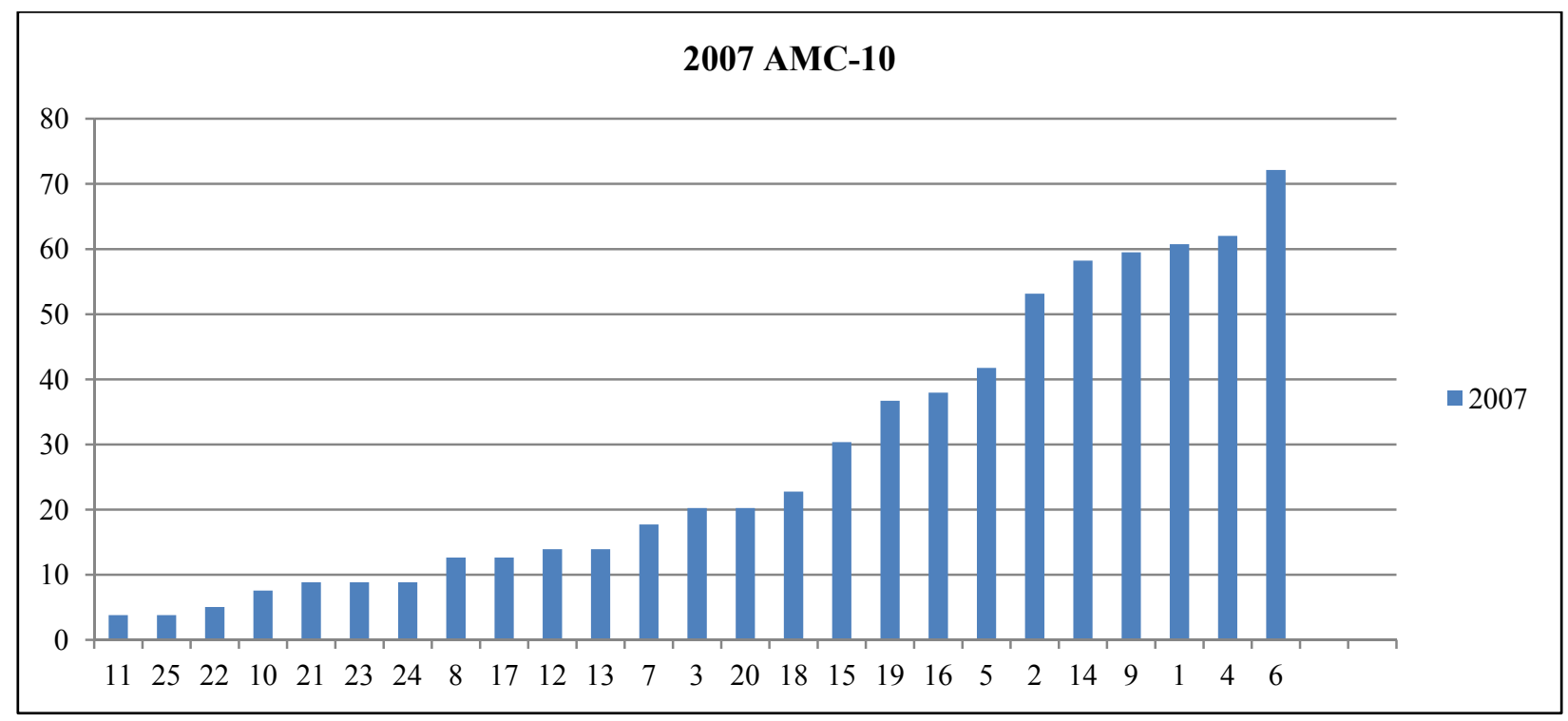

Figure 2. Performance of students on AMC-10 in 2007.

Table 5a

Student Performance on AMC-10 in 2007

\begin{tabular}{|c|c|}
\hline Proficiency & Deficiency \\
\hline \multicolumn{2}{|l|}{ 6. Algebra-inequalities $(72.15 \%)$} \\
\hline 4. Geometry - interior angle of a triangle inscribed in a circle & 3. Algebra—distance-speed-time/averages $(20.25 \%)$ \\
\hline 1. Algebra/Geometry - areas of rectangular objects $(60.76 \%)$. & 7. Geometry—convex pentagon/angle measure $(17.72 \%)$. \\
\hline 9. Discrete structures - counting principles $(59.49 \%)$. & 8. Discrete Structures—counting principles $(12.66 \%)$. \\
\hline 14. Algebra-percentages $(58.23 \%)$. & 16. Algebra—percentages/averages (37.97\%). \\
\hline 2. Algebra-function operation/relation (53.16\%). & 17. Geometry-equilateral triangles/perpendiculars $(12.66 \%)$. \\
\hline 5. Logic - conditional proposition "if ..., then ..." (41.77\%). & \\
\hline
\end{tabular}

On the 2007 AMC-10 test, the students showed a reasonable degree of proficiency (at least $40 \%$ ) on several topics as can be seen from Table 5a above. The seven problems in which students' performance was good were all of regular strength. The students did not perform well on five problems of similar strength. Perhaps, the type of application made the difference rather than the topic. In sum, this group had a 58.3\% success rate in 2007. In the proficiency column in Table 5a, we list the problems and name the topics in descending order of students' performance. However, problems and their respective topics in the deficiency column were listed serially (with no regard to performance levels).

In the sequel, we list areas of proficiency and areas of deficiency similar to the above description. In addition, we give the rate of success for each year (see Tables $5 \mathrm{~b}-5 \mathrm{e}$ ).

Table $5 \mathrm{~b}$

Student Performance on AMC-10 in 2008 (Success Rate: 60\%)

\begin{tabular}{|c|c|}
\hline Proficiency & Deficiency \\
\hline \multicolumn{2}{|l|}{ 1. Discrete structures - counting $(88.04 \%)$. } \\
\hline 12. Algebra-distance-rate-time $(72.83 \%)$. & 3. Algebra—roots/exponents $(22.83 \%)$. \\
\hline 2. Discrete structures - counting $(67.39 \%)$. & 5. Algebra-function/relation (31.52\%). \\
\hline 4. Algebra-ratio/proportion (64.13\%). & 9. Algebra-quadratic equation $(13.04 \%)$. \\
\hline 7. Geometry-equilateral triangles $(54.35 \%)$. & 10. Geometry — circles/lines $(11.96 \%)$ \\
\hline 8. Algebra—ratio/proportion (46.74\%). & \\
\hline
\end{tabular}


Table 5c

Student Performance on AMC-10 in 2009 (Success Rate: 66.7\%)

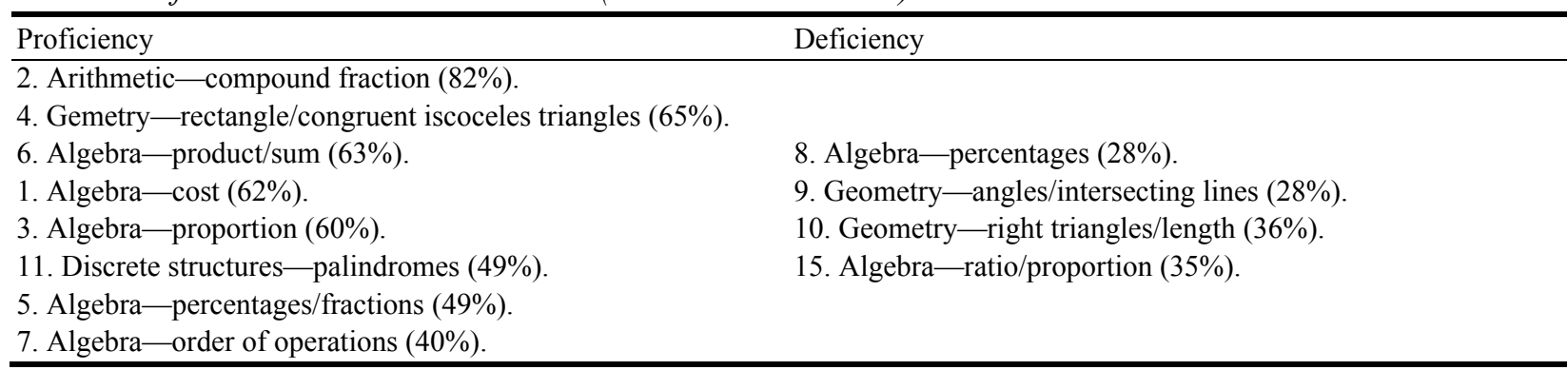

Table 5d

Student Performance on AMC-10 in 2010 (Success Rate: 50\%)

\begin{tabular}{|c|c|}
\hline Proficiency & Deficiency \\
\hline 2. Algebra-fractions/percentages (80\%). & 4. Algebra-relations (38\%). \\
\hline 1. Algebra-order of operations $(79 \%)$. & 5. Discrete structures - counting (37\%). \\
\hline 7. Geometry—rectangle/isosceles triangle/area (55\%). & 8. Algebra—divisors/multiples $(27 \%)$ \\
\hline 6. Geometry—circle/angle measure $(55 \%)$ & 9. Algebra- order of operations $(37 \%)$. \\
\hline 3. Discrete Structures - counting (51\%). & 10. Algebra-distance-rate-time (31\%). \\
\hline 15. Discrete Structures-counting (44\%). & 12. Algebra-ratio/percentages (14\%). \\
\hline
\end{tabular}

Table 5e

Student Performance on AMC-10 in 2011 (Success Rate: 41.7\%)

\begin{tabular}{|c|c|}
\hline Proficiency & Deficiency \\
\hline $\begin{array}{l}\text { 2. Algebra-averages/minimum-maximum }(78 \%) \text {. } \\
\text { 1. Algebra-fraction addition }(76 \%) \text {. } \\
\text { 3. Geometry-rectangle/minimum area }(60 \%) \text {. } \\
\text { 6. Algebra-fraction application }(52 \%) \text {. } \\
\text { 8. Logic-"if ..., then ..." }(50 \%) \text {. }\end{array}$ & $\begin{array}{l}\text { 4. Algebra-fraction application }(34 \%) \text {. } \\
\text { 5. Algebra-numbers/digits/product }(29 \%) \text {. } \\
\text { 7. Geometry-angles/fractions }(31 \%) \text {. } \\
\text { 10. Algebra-ratios }(16 \%) \text {. } \\
\text { 11. Discrete structures-counting }(7 \%) \text {. } \\
\text { 15. Algebra-defined relation }(14 \%) \text {. } \\
\text { 18. Geometry-rectangle/angle measure }(7 \%) \text {. }\end{array}$ \\
\hline
\end{tabular}

During the five years (i.e., 2007-2011) of interest, students taking the AMC-10 tests performed at proficiency rates of $58.3 \%, 60 \%, 66.7 \%, 50 \%$, and $41.7 \%$, respectively. The approximate range of their success rate was $42 \%-67 \%$ with an average of $55 \%$. In effect, students were deficient on approximately $45 \%$ of problems that are a part of the regular high school curriculum.

\section{Students' Performance on the AMC-12 Tests}

As with the AMC-10 tests, we now present a summary of the five-year performance of students on the AMC-12 tests. Figure 3 gives the percentages of correct responses for each of the 25 questions for each year.

Next, we examine the data for each year to identify problems and topics on which at least $40 \%$ of students provided correct responses. As before, we assess the students' performance relative to the minimum $40 \%$ benchmark (see Figure 4). We also identify problems and topics on which the students did not meet the $40 \%$ bar and classify the attendant topic or problem as belonging to an area of deficiency for the group of students that took the test. Furthermore, we isolate those problems that were also found on the AMC-10 test for the corresponding year. The students' success rate on those "repeat" problems are also given. 


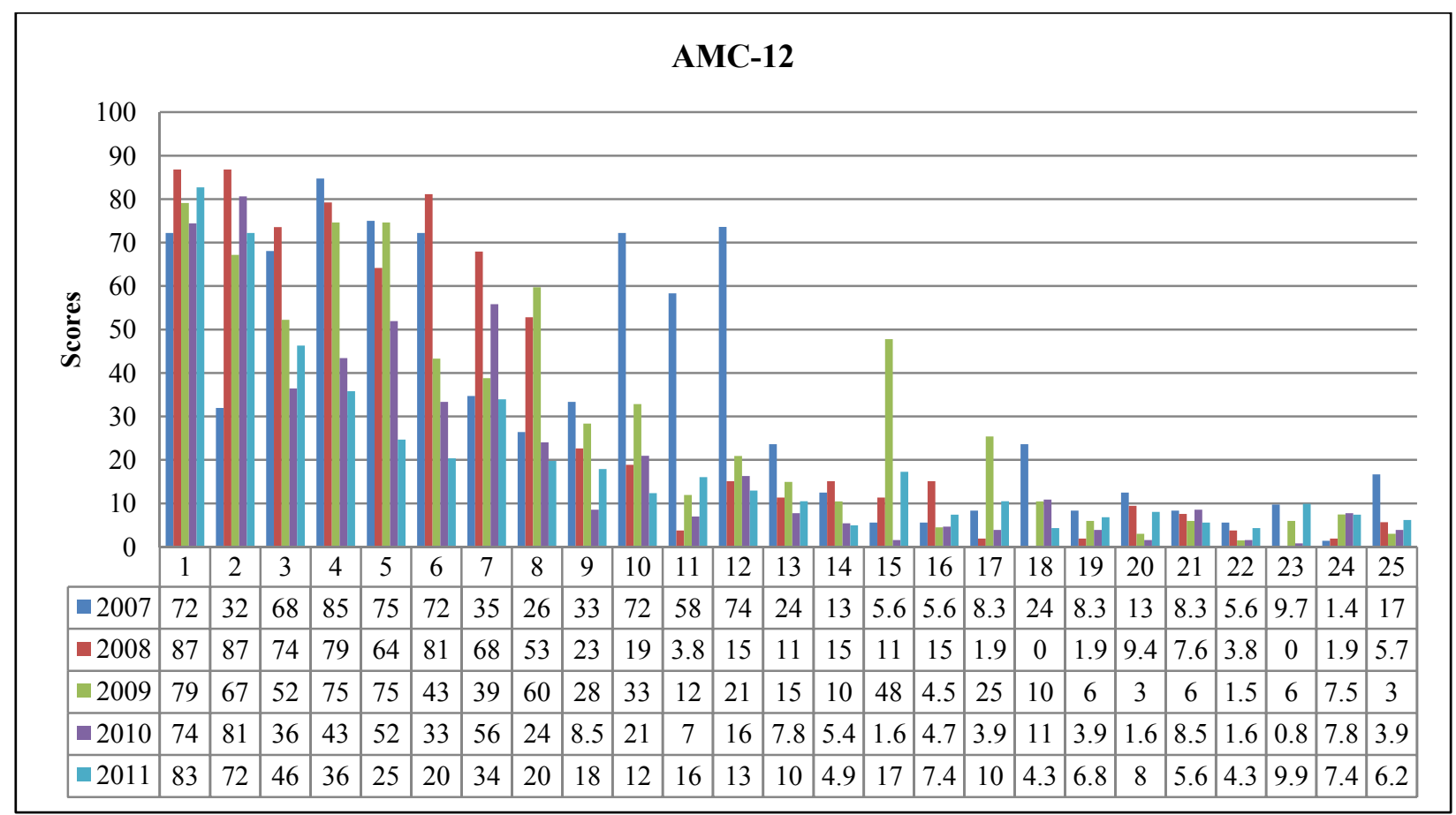

Figure 3. Percentages of correct responses.

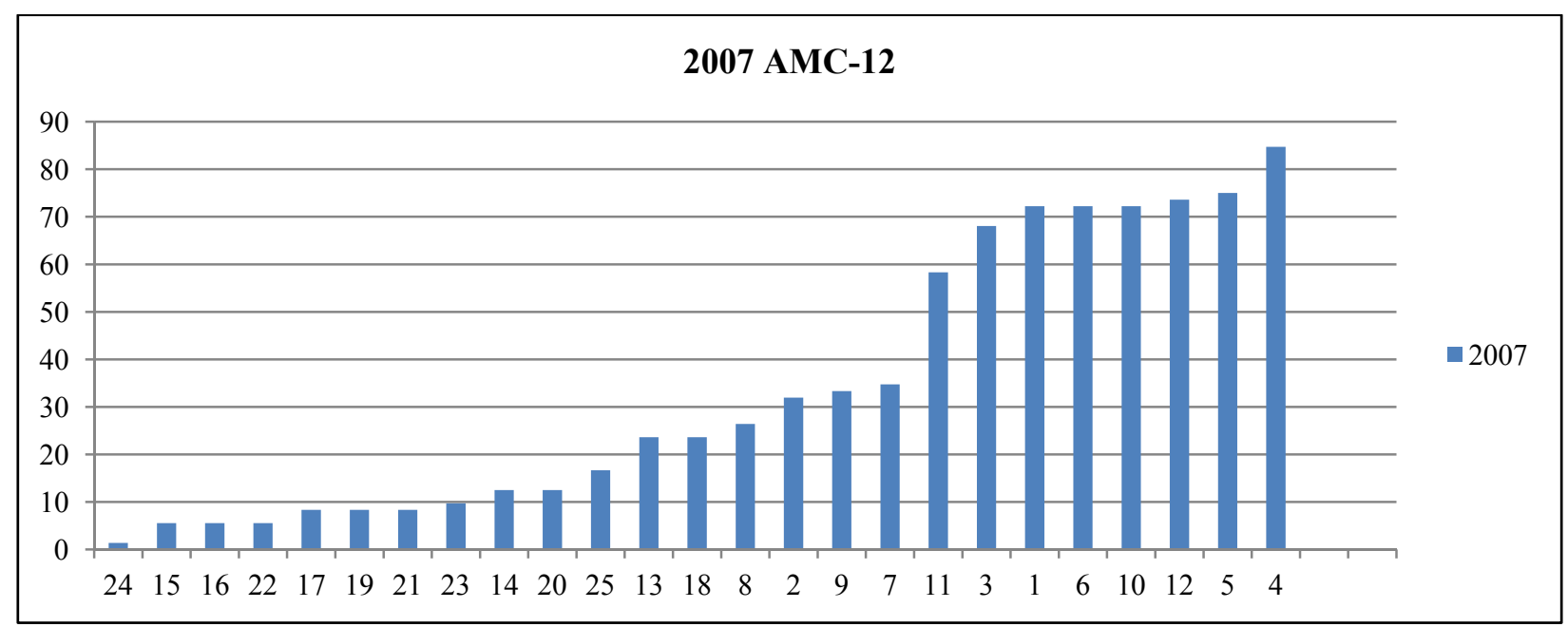

Figure 4. Performance of students on AMC-12 2007.

Table 6 a

Students' Performance on AMC-12 in 2007 (Success Rate: 60\%; Repeats: 70\%)

\begin{tabular}{|c|c|}
\hline Proficiency & Deficiency \\
\hline $\begin{array}{l}\text { 4. Algebra-ratio/proportion }(85 \%) \text {. } \\
\text { 5. Algebra-ratio/inequalities }(75 \%) \text {. } \\
\text { 12. Algebra-percentages }(74 \%) \text {. } \\
\text { 10. Algebra-percentages }(72 \%) \text {. } \\
\text { 6. Geometry - triangles/perimeter }(72 \%) \text {. } \\
\text { 1. Algebra/Geometry-rectangles/area }(72 \%) \text {. } \\
\text { 3. Geometry - circle/inscribed triangle }(68 \%) \text {. } \\
\text { 11. Geometry-quadrilateral/angle }(58 \%) \text {. }\end{array}$ & $\begin{array}{l}\text { 2. Algebra-average distance/time/rate }(32 \%) \text {. } \\
\text { 8. Algebra-application of addition }(26 \%) \text {. } \\
\text { 9. Algebra-function operation }(33 \%) \text {. }\end{array}$ \\
\hline
\end{tabular}


The success rate on regular strength problems was $60 \%$. The group was proficient on one medium strength problem (6). In addition, they were proficient on $70 \%$ of the "repeat" problems. That is, problems also found on the AMC-10 test for the same year.

Table $6 \mathrm{~b}$

Performance on AMC-12 in 2008 (Success Rate: 72\%; Repeats: 55\%)

\begin{tabular}{|c|c|}
\hline Proficiency & Deficiency \\
\hline $\begin{array}{l}\text { 2. Discrete structures-counting }(87 \%) \text {. } \\
\text { 1. Discrete structures - counting }(87 \%) \text {. } \\
\text { 6. Algebra-distance/rate/time }(81 \%) \text {. } \\
\text { 4. Algebra/Geometry-circle/ratio }(79 \%) \text {. } \\
\text { 3. Algebra - fraction application }(74 \%) \text {. } \\
\text { 7. Algebra-defined relation }(68 \%) \text {. } \\
\text { 5. Algebra-ratio/fraction }(64) \text {. } \\
\text { 8. Algebra-proportion }(52 \%) \text {. }\end{array}$ & $\begin{array}{l}\text { 9. Geometry—circle/line-midpoint/minor/length }(22 \%) \text {. } \\
\text { 10. Algebra-work done. } \\
\text { 11. Geometry-cone/volume. }\end{array}$ \\
\hline
\end{tabular}

Table $6 \mathrm{c}$

Performance on AMC-12 in 2009 (Success Rate: 78\%; Repeats: 60\%)

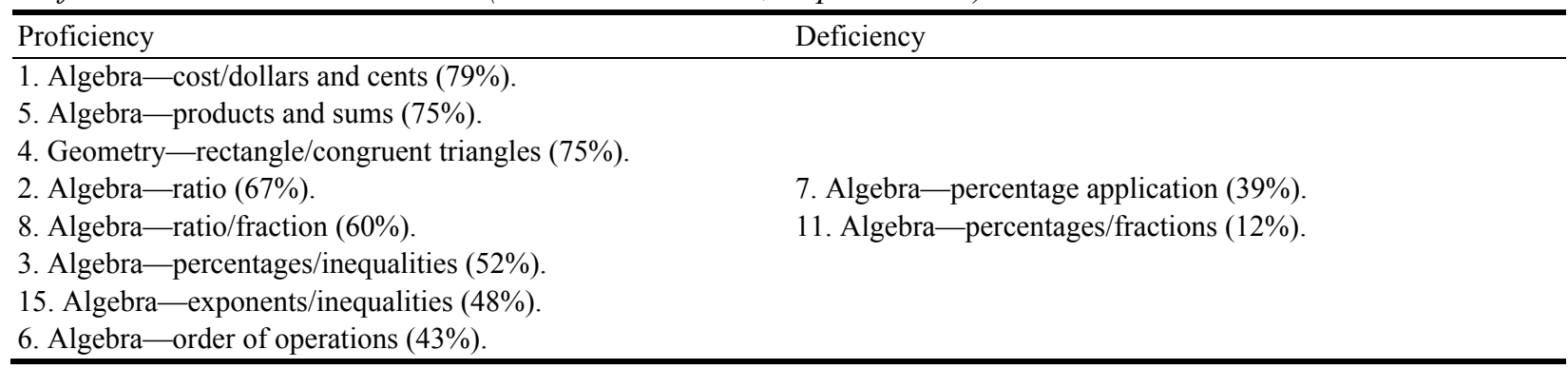

Table 6d

Students' Performance on AMC-12 in 2010 (Success Rate: 56\%; Repeats: 31\%)

\begin{tabular}{|c|c|}
\hline Proficiency & Deficiency \\
\hline $\begin{array}{l}\text { 2. Geometry-rectangle/ntages.area }(81 \%) \text {. } \\
\text { 1. Algebra-percentages }(74 \%) \text {. } \\
\text { 7. Algebra-distance/rate/time/averages }(56 \%) \text {. } \\
\text { 5. Algebra-order of operations }(52 \%) \text {. } \\
\text { 4. Discrete structures - counting }(43 \%) \text {. }\end{array}$ & $\begin{array}{l}\text { 3. Algebra-cost/dollars and cents }(36 \%) \text {. } \\
\text { 6. Algebra-percentage application }(33 \%) \text {. } \\
\text { 8. Discrete structures - counting }(24 \%) \text {. } \\
\text { 10. Discrete structures - counting }(21 \%) \text {. }\end{array}$ \\
\hline
\end{tabular}

Table 6e

Students' Performance on AMC-12 in 2011 (Succes Rate: 43\%; Repeats: 27\%)

\begin{tabular}{|c|c|}
\hline Proficiency & Deficiency \\
\hline $\begin{array}{l}\text { 1. Algebra-fraction addition }(83 \%) \text {. } \\
\text { 2. Algebra-averages }(72 \%) \text {. } \\
\text { 3. Algebra-fraction/average }(46 \%) \text {. }\end{array}$ & $\begin{array}{l}\text { 4. Algebra-product/sum/digits }(36 \%) \text {. } \\
\text { 5. Algebra-least commom multiple }(25 \%) \text {. } \\
\text { 10. Geometry-rectangle/angle measure }(12 \%) \text {. } \\
\text { 11. Geometry-coordinates/distances }(16 \%) \text {. }\end{array}$ \\
\hline
\end{tabular}

For the five-year period, the various groups of students who took the AMC-12 tests had an average success rate of $61.8 \%$ on the regular strength problems and $48.5 \%$ on the problems that were also on the AMC-10 tests (see Tables 6a-6e).

\section{Students' College Readiness for Mathematics}

In their published study, Li, Uvah, Amin, and Okafor (2010) associated students' performance in College 
Algebra with their readiness for college mathematics. Based on the reasonable argument that they advanced in order to draw that conclusion, we elected to identify problems on the AMC of the types that are to be found in College Algebra. The UWF student learning outcomes for College Algebra are as follows:

1. Identifying (algebraic and transcendental) functions and their properties;

2. Analyzing and graphing polynomials, rational, radical, exponential, and logarithmic functions;

3. Performing operations on algebraic and transcendental functions;

4. Solving exponential and logarithmic equations, and systems of linear equations;

5. Solving problems involving applications of algebraic and transcendental functions.

Problems on the AMC tests that assess the students' abilities with respect to the above learning outcomes for College Algebra were of regular and medium strength with the vast majority being of regular strength. Consequently, we targeted the regular strength algebra problems on the AMC-12 exclusively, to assess the college readiness of the 11th and 12th grades students. In Table 7 below, we give a summary of the students' performance on the identified algebra questions. As before, we considered the group to be deficient on a topic if less than $40 \%$ of the students provided accurate responses.

Table 7

Students' Performance in AMC-12 on Algebra Topics (2007-2011)

\begin{tabular}{ll}
\hline Proficiency & Deficiency \\
\hline Order of operations, products, and sums & Fraction addition application \\
Fraction addition, fraction application & Product, sum, and digits \\
Averages of numbers & Least commom multiple \\
Percentages & Cost, dollars, and cents \\
Percentages and inequalities & Percentage and ratio application \\
Ratio and proportion & Percentages and fractions \\
Ratio and inequalities & Straight line and midpoint \\
Distance-rate-timedirect application & Ratio and work done \\
Defined relation operation & Averages with distance-time-rate \\
Cost and systems of equations & Defined function operation \\
Exponents and inequalities & Exponential function operation \\
\hline
\end{tabular}

As Table 7 shows, the 11th and 12th grades students who took the AMC-12 (2007-2011) did reasonably well on approximately $50 \%$ of the algebra topics tested, overall. However, a closer examination shows that the algebra questions on which at least $40 \%$ of them provided correct answers were less than $45 \%$ in any given year. That is, their success rate on the algebra questions was below $45 \%$ each year. The weaknesses that the group showed spanned the spectrum of the college algebra student learning outcomes named above. Topics that presented the most severe issues included: solving, interpreting or applying exponential and logarithmic functions and equations; performing operations on transcendental functions; and solving problems involving algebraic and transcendental functions. We concluded that $35 \%-40 \%$ of them showed college readiness for College Algebra. As can be seen in Table 2, most of the 2009 group of students had taken mathematics courses beyond the required Algebra I and Geometry in spite of the poor group performance.

\section{Factors That Affect Students' Performance in Mathematics}

Although the factors that affect students' performance are varied and may differ from one place to the other, a few of these factors are universal and well-known. These include: 
1. Teacher preparation — ideally, teachers with state and/or national certification, and a master's degree in mathematics are considered to be well-prepared;

2. Economic situation - here, demographical indices concerning families are used to determine if a school district is a "high need" one. Such indices include: rates of free and reduced lunch at school, other Title I programs, and high rates of African American or ethnic minorities in the student body;

3. Curriculum matters - non-uniformity of course offerings for a given grade-level in middle and high schools across a school district slows down a given class in the 9th and subsequent grades;

4. Low expectation - teachers' expectation of students, like the districts' expectations of teachers and the state's expectation of districts, all have a well-known effect on students' performance. State requirements for high school graduation and locally determined guidelines for promoting students from one grade level to the next, are good indicators of the system's expectations of their students.

We note in passing, that each of the factors listed above was present in the two counties from which students that took part in the AMC tests. See, for example, the report by The UWF statistical consulting center (2007).

\section{Conclusion}

\section{Summary}

In order to assess the mathematics proficiency among high school students in Northwest Florida, we collected data on 970 students from the school districts of Escambia and Santa Rosa who took the AMC tests at the UWF test center from 2007 to 2011. There were 483 students, 11th and 12th graders, who took the AMC-12 while 487 (9th and 10th graders) took the AMC-10 tests. We first coded the problems on each test according the level of difficulty in order to identify those that were similar to problems that are discussed in a regular classroom setting. Students' group performance on the regular strength problems was analyzed such that a group was deemed to be proficient on a given problem if at least $40 \%$ of students in the group gave correct responses to the question in the problem. From the examination and analyses:

Most of the test questions in the AMC-10 tests for 9th and 10th grades students' were from topics in Algebra and Geometry. However, there were also a few questions on counting principles in discrete mathematics that demanded a commonplace understanding rather than an analytical method of solution. On the regular strength questions that we isolated and analyzed, the students performed at an average proficiency rate of $55 \%$. Thus, the students as a group were seen to be deficient on $45 \%$ of the material that is usually covered in their classes.

For the 11th and 12th grades students who took the AMC-12 tests, group performance on regular strength problems was at an average proficiency rate of $62 \%$. The higher rate in comparison to the group who took the AMC-10 tests was not surprising since our survey showed that many of the 11th and 12th graders took some mathematics courses beyond Algebra I and Geometry. Among other things, we deduce from this higher performance on the regular strength problems, that students who are exposed to higher levels of mathematics will have a better understanding of lower-level mathematics.

Approximately, 12 problems of regular and medium strength appeared on both tests for each of the years of interest in our study. It was striking, however, that the average performance rate of those taking the AMC-12 on the repeated questions (most of which were of regular strength) was only $49 \%$. That is, the students taking the AMC-12 provided incorrect answers on over half of the questions that were repeated on the tests. 
Using the student learning outcomes for College Algebra and well-known studies on college readiness, it was evident that a meager $35 \%-40 \%$ of the 11 th and 12 th grades students were ready for college-level mathematics courses. This result was surprising because our survey showed that approximately $70 \%$ of the 11 th and 12th grades students who took the AMC-12 tests in 2009 had taken several mathematics courses beyond the Algebra I and Geometry that formed the mathematics requirement for high school graduation.

\section{Recommendations}

The following recommendations emanate from our cumulative experiences with the state and the school districts (Florida Department of Education [FDOE], 2007). The analyses of students' performance on the AMC tests have further crystallized some of the hypotheses that we had formed before this study.

School districts should dictate uniform standards for grade levels beginning with the 6th grade in middle school. A unified syllabus for each course with weekly or biweekly topics to be covered and minimum exercises for class and homework will give teachers and parents good guidance regarding school district expectations and standards. Students who have cause to change schools during the school year or at the completion of a grade should be starting in a new school at a well-known point in their mathematics curriculum. This may also help with appropriate placement into higher mathematics courses in the 9th grade.

The State of Florida has recently raised the minimum requirement in mathematics for high school graduation to include Algebra II. The statewide Florida Comprehensive Assessment Test (FCAT) is also being replaced by district-wide end-of-year tests (ENLACE-Florida, 2009). School districts can build on this new order, to institute higher expectations by dictating stricter standards that are over and above the state minimum requirements. Students who have problems passing any of these courses can be further helped to achieve the expected higher levels while they are in the 11th grade but should be made to take an elective but higher-level mathematics course in the 12 th grade.

Each school district should assess students' group performance for the district and for each school, year after year. With a unified syllabus and one end-of-year district examination, data on the district examination should be collected and used to assess students' performance, to identify deficiencies by topic. Districts should require schools to use the assessment results to develop mitigation plans to include: spending more time on areas of deficiency; shifting appropriate parts of the deficiency to be introduced in an earlier or future course; revising the minimum exercises for a topic of deficiency; or providing help outside the classroom setting. Mitigation efforts that produce excellent results should be publicized among "good practices" to be widely accessed throughout the district for greater impact.

There is a real need to significantly step up teacher preparation for STEM areas, especially mathematics. School districts should make long-term investment in STEM by supporting STEM teachers towards state and national certification, higher degrees, workshops, symposia, and other faculty development activities. Collaborative efforts between district teachers and regional universities and colleges will help to place teachers on a sound footing, so that they are current on the content that they teach and have a variety of hands-on projects to motivate their students.

The state and school districts may be able to attract brilliant teachers in STEM if they provide scholarships and fellowships, not loans, to good college students who could be contracted to teach for a specified minimum number of years after their graduation. Similarly, steps should be taken to arrest the roadblock whereby STEM graduates who opt to teach are saddled with certification procedures that are extremely bureaucratic and 
convoluted. Good STEM graduates often have other career options available to them and are needlessly discouraged by cumbersome certification processes. Moreover, schools are now routinely requiring STEM teachers to also coach school teams or partake in extra-curricular activities that are not directly related to teaching. Additional teachers' responsibilities should be aligned with programs and curricula, so that the teachers also see the link between those activities and the courses that they teach.

Schools should create and encourage more co-curricular activities that are STEM-based with hands-on activities for students, so that many students will have a better appreciation for the sciences at an early age. Student organizations and clubs that provide visibility are an excellent means of providing motivation and interest in the sciences. Of course, aside from institutional and district support, there is a need for teachers with creative ideas to act as sponsors and advisors, yet another reason for districts to engage in enhanced faculty development activities that give the teachers a set of activities from which to choose.

The idea of employing STEM-trained personnel to shoulder the responsibility of academic guidance tailored to STEM is overdue. Such counselors may readily identify, place, and mentor students who have shown exceptional interest in STEM. Moreover, a STEM counselor could coordinate some of the co-curricular activities and craft avenues for parents' involvement and education regarding the identified interests of their children.

The word-problem format of the AMC tests appeared to be a contributing factor in the poor performance of students, especially on relatively simple problems with lengthy wording. A conscious effort should be made to subject students to word problems involving the mathematics concepts that they are learning, beginning from the middle school grades. Given that word problems bring together a student's utilization of reading, analytical and symbolic manipulation skills, universities and school districts should collaborate on professional development workshops for teachers, so as to successfully implement a word-problem scheme for mathematics.

As the above analysis shows, a majority of the 11th and 12th grades students who took the AMC-12 tests did not show college readiness for mathematics. Moreover, studies, such as Stumpf and Stanley (2002), Allen and Sconing (2005), and Li, Uvah, Amin, and Okafor (2010) suggested that scores on standardized tests might not be a reliable indicator of performance in College Algebra. A divergent opinion can be found in the College Board Report (2008). Consequently, we suggest that colleges should develop and administer a placement test to be used as an additional tool for placing students in the most appropriate college-level mathematics course. Students who begin their college career at a comfortable level in mathematics are more likely to succeed and develop interest in the STEM areas.

The State of Florida and universities should consider introducing some of the topics that are currently taught only in College Algebra in a preparatory course, such as Intermediate Algebra. In particular, topics in which students continue to do poorly in College Algebra should be introduced earlier, to include: piece-wise, logarithmic, and exponential functions; equations involving these and rational functions; and at a minimum, a graphical treatment of domain and range of functions.

In order to generate further interest in STEM, college admissions and financial aid offices should recognize participation and performance in co-curricular activities, such as the AMC tests.

\section{References}

Allen, J., \& Sconing, J. (2005). Using American College Test (ACT) assessment scores to set benchmarks for college readiness. ACT Report Series, 3, 1-24. 
Engaging Latino Communities in Education (ENLACE) Florida. (2009). Opening the gates to success in Florida higher education. ENLACE Publication, 3(5), 1-10.

Florida Department of Education (FDOE). (2007). College and career readiness standards and assessments. Tallahassee, F.L.: Florida Department of Education. Retrieved July 28, 2011, from http://www.fldoe.org/cc/gohigher/2007_0430/Davecollegereadiness.pdf

Li, K., Uvah, J., Amin, R., \& Okafor A. (2010). A study of college readiness for College Algebra. Journal of Mathematical Sciences \& Mathematics Education, 5(1), 52-66.

Stumpf, H., \& Stanley, C. J. (2002). Group data on high school grade point averages and scores on academic aptitude tests as predictors of institutional graduation rates. Educational and Psychological Measurements, 62(6), 1042-1052.

The College Board Report. (2008). Validity of the Scholastic Aptitude Test (SAT) for predicting first-year college grade point averages. Retrieved July 26, 2011, from http://professionals.collegeboard.com/profdownload/Validity_of_the_SAT_for_ Predicting_First_Year_College_Grade_Point Average.pdf

The UWF Statistical Consulting Center. (2007). Escambia county, the 2004-2005 No Child Left Behind reports for K-12 institutions. Retrieved July 2011, from http:/uwf.edu/cse/departments/mathematics-and-statistics/research/technical-reports/ 